\title{
Commentary: How to transpose the isolated left vertebral artery in hybrid thoracic endovascular aortic repair
}

Tohru Asai, MD, PhD

\author{
From the Department of Cardiovascular Surgery, Juntendo University, Tokyo, Japan. \\ Disclosures: Author has nothing to disclose with regard to commercial support. \\ Received for publication June 17, 2019; accepted for publication June 17, 2019; available ahead of print July 30 , \\ 2019. \\ Address for reprints: Tohru Asai, MD, PhD, Department of Cardiovascular Surgery, Juntendo University, Faculty \\ of Medicine, 2-1-1 Hongo, Bunkyo-ku, Tokyo 113-8421, Japan (E-mail: t.asai.jj@juntendo.ac.jp). \\ J Thorac Cardiovasc Surg 2020;159:2199 \\ $0022-5223 / \$ 36.00$ \\ Copyright (C) 2019 by The American Association for Thoracic Surgery \\ https://doi.org/10.1016/j.jtcvs.2019.06.068
}

In this issue of The Journal of Thoracic and Cardiovascular Surgery, Piffaretti and colleagues ${ }^{1}$ presented their experience of managing the isolated left vertebral artery (ILVA) during hybrid aortic arch repair with thoracic endovascular aortic repair (TEVAR) completion. The ILVA arises directly from the aortic arch, usually between the left common carotid artery and the left subclavian artery, and it is the second most common anatomic variant of the supra-aortic configuration. Although their study is a single-center, observational experience, management of ILVA can be a critical arch repair, depending on the specific conditions in each case. Out of 200 patients in their TEVAR experience, 9 $(4.5 \%)$ were found to have ILVA. The specific strategy of Piffaretti and colleagues ${ }^{1}$ was to transpose the ILVA in all cases, and they claim that this is feasible, durable, and appropriate in most cases.

Cerebrospinal complications after thoracic aortic surgery are nightmarish. Any atheromatous embolism or small vessel occlusion could cause dreadful morbidity in patients who were clearly awake preoperatively. For this specific issue of ILVA, there are no clear guidelines because of a lack of data. The overall quality of published evidence is low. Moreover, in the anatomy of vertebral artery blood flow, dominance and patterns vary. ${ }^{2}$ In some cases, right and left vertebral arteries of similar diameter connect to the basilar artery, but the right and the left vertebral arteries usually have different diameters in connected cases. There are also cases in which the right or the left vertebral artery is interrupted and not connected to the basilar artery. The posterior inferior cerebellar artery may be supplied by the interrupted nondominant vertebral artery. All these variations need to be considered when surgeons plan aortic arch repair.

In their complex hybrid repair strategy, Piffaretti and colleagues ${ }^{1}$ successfully performed ILVA transposition in issue related to cerebrospinal complications after aortic

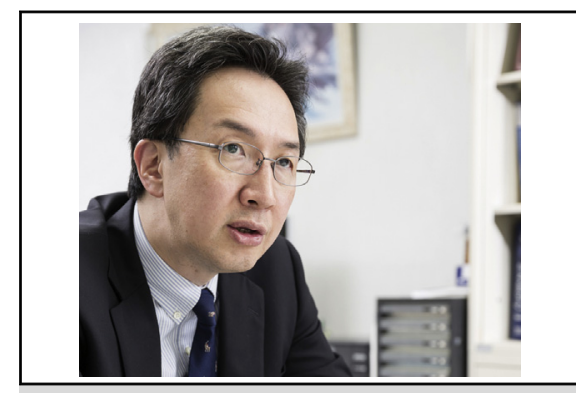

Tohru Asai, MD, PhD

Central Message

Transposition of the isolated left vertebral artery may be performed safely, and is durable when it is necessary.

See Article page 2189. various ways. Their outcomes were excellent in most of the life-threatening aortic arch cases in hybrid TEVAR, except in 1 case of a patient with Horner syndrome. In their series, there was no thrombosis in any transposed ILVA, no local nerve palsy, and no chylothorax. Nor were any postoperative cerebrovascular accidents or spinal cord injuries observed. Piffaretti and colleagues ${ }^{1}$ conclude that ILVA transposition is feasible, may be performed safely, and is durable, both during open ascending arch repair and during isolated "zone 2" TEVAR.

There are still few reported series focusing on ILVA during aortic arch repair. I hope that the article of Piffaretti and colleagues ${ }^{1}$ will draw the attention of our readers to this alltoo-possible anatomic variation and will encourage them to collect adequate amounts of relevant data to guide us better to understand the risks of complications, to construct future evidence-based guidelines for decision making appropriate to every patient.

\section{References}

1. Piffaretti G, Gelpi G, Tadiello M, Ferrarese S, Socrate AM, Tozzi M, et al. Trans position of the left vertebral artery during endovascular stent-graft repair of the aortic arch. J Thorac Cardiovasc Surg. 2020;159:2189-98.e1.

2. Ohkura K, Shiiya N, Washiyama N, Yamashita K, Takahashi D, Tsuda K, et al Vertebral artery variations in thoracic aortic patients. Eur J Cardiothorac Surg. 2014;46:27-31. 